\title{
Determinants of Dietary Diversity among Pregnant Women Attending Public Health Facilities in Hossana town,South Ethiopia
}

Degefa Helamo Kobiro ( $\nabla$ degefa.helamo@gmail.com )

Hossana college of health sciences

Romedan Delil

Hossana college of health sciences

Desalegn Tamiru

Jimma University

Beakal Zinab

Jimma University

Research article

Keywords: Dietary diversity, Determinants, Pregnancy

Posted Date: December 4th, 2019

DOI: https://doi.org/10.21203/rs.2.11374/v2

License: (1) This work is licensed under a Creative Commons Attribution 4.0 International License.

Read Full License 


\section{Abstract}

Background: Dietary diversity refers to increasing the consumption of a variety of foods across and within the food groups, adequate and diversified diets during pregnancy is essential for fetus health, body maintenance and enhancement of maternal health through protection of infectious and noncommunicable diseases. Hence, this study was targets to assess dietary diversity and its determinants among pregnant women of public health facilities in Hossana town, south Ethiopia Methods: Facility based cross-sectional study was applied among selected 303 pregnant women in Hossana town from June 1 to July 15, 2018. Systematic random sampling procedure was employed to recruit study participants. Data were collected using an interviewer administered pretested semi-structured questionnaire, one week dietary consumption was used to compute women dietary diversity score. Descriptive statistics and multivariable logistic regression were done to isolate predictors of dietary diversity. Result: The prevalence of low, medium and high women's dietary diversity was 9.2, 48.2 and $42.6 \%$ respectively. Educational status ( $\mathrm{AOR}=2.1 ; 95 \% \mathrm{Cl}: 4.8,15.2)$, monthly income ( $\mathrm{AOR}=2.1 ; 95 \% \mathrm{Cl}$ : 4.8, 15.2), nutritional education ( $\mathrm{AOR}=2.3 ; 95 \% \mathrm{Cl}$ : $1.2,4.4)$ and frequency of eating ( $\mathrm{AOR}=12.9 ; 95 \% \mathrm{Cl}$ : $3.1,52$ ) were meaningfully associated with women dietary diversity. Conclusions: In this study women educational status, monthly income, receiving nutritional education and frequency of eating were determinants of women dietary diversity. Efforts should be strengthened towards improving women educational level, delivery of nutritional education and enhance frequency of eating among pregnant women to advance dietary diversity. Keywords: Dietary diversity, Determinants, Pregnancy

\section{Background}

Adequate diversified diets are essential for fetus health, body maintenance and enhancement of maternal health through protection of infectious and non-communicable diseases globally [1]. Dietary diversity refers to increasing the consumption of a variety of food across and within the food groups; it has been recognized as a crucial indicator for surveillance of actions that aim to challenge various nutrition-related problems and food insecurity among pregnant women in the developed and developing world [2]. In resource-poor environments across the world, low quality monotonous diets are the habit; when grain or tuber-based staple foods dominate and diets lack vegetables, fruits and animal source foods, micronutrient deficiencies is high; whereas information on micronutrient deficiencies is scarce, it is clear that poor micronutrient status among women is a global problem and is most severe for poor women, information about dietary patterns and diet quality for women across countries is limited [3]; inadequate food intake, poor nutritional quality of diets, frequent infections and short inter-pregnancy intervals are among the most common immediate causes of maternal and newborn malnutrition [4]. Nutrient deficiencies and imbalanced dietary intake tend to occur during the reproductive period in developed and developing countries of pregnant women in urban and rural areas [5], intake of different variety of foods has been an endorsement of achieving adequate nutrient intake and the recommendation appears the dietary diversity guideline of various countries[6].Maternal nutritional requirement of macro and micronutrients increase during pregnancy meeting these requirements is crucial for the appropriate 
health, growth, development of fetus and women healthy life [7], healthy eating practices and consumption of food from variety of dietary groups during pregnancy affects fetal health and contributes to prevention of pregnancy-related complications [8]. Among food groups ingesting cereals, wheat, pulses, roots and tubers are the major component of the diet in most part of Africa [9]. Ethiopia is among the poor country in Africa with high rates of food insecurity, low-quality nutrients, monotonous diets and malnutrition; moreover, diet diversity among pregnant was significantly lower than non-pregnant women in south Ethiopia more than half of women avoided at least one food type during their pregnancy [10, 11].Pregnancy is the utmost nutritionally demanding period in a woman's life; thus, pregnant women are advised to eat more diversified diet than usual; consumption of meat, fish, fruits and some vegetables during pregnancy despite some region remained lower than the pre-pregnancy period, a number of food misconceptions related to consumption of green leafy vegetables, yogurt, cheese, sugar cane and green pepper food items adversely affect nutritional status during pregnancy in Ethiopia [12].Household dietary diversity score in south part Ethiopia is five, cereals are the most usually consumed food groups; in contrast fish, egg and fruits are the least consumed. Animal source foods are eaten in greater amount among families with higher dietary diversity, identifying nutritional status individual members of the family important for new technologies supporting agricultural practices to enhance both native production and improved consumption diversified diets of pregnant women in Hadiya Zone [10].The objective of this study was to assess dietary diversity and its determinants among pregnant women of public facilities in Hossana town, south Ethiopia and it might be used among all women of childbirth age, having data on low dietary diversity and less variety diet consumption is a proxy of low quality diet, nutritional status and food insecure among pregnant women in the town.

\section{Methods}

\section{Study area and period}

Facility based cross sectional study was applied in all public health facilities of Hossana town from June 1 to July $15,2018$.

\section{Source and study population}

Source population were all pregnant women attending Antenatal care (ANC) at Wachamo Negist Eleni Memorial Referral Hospital; Lichamba, Bobicho and Hossana Health Centers which are all public health facilities of Hossana town but study population pregnant women who has follow up during data collection and study participants were randomly selected pregnant women attending ANC at public health facilities of the town during the study and who met the inclusion criteria; over-all 303 pregnant were recruited in this study who had residents of at least six month in the town; pregnant women who had special dietary recommendations due to other morbidities or chronic diseases were excluded from the study.

\section{Sample size and sampling technique}


The sample size was calculated using single population proportion formula based on the following assumptions proportion of dietary diversity of $34.3 \%$ [13] reported from the previous study and using the $95 \%$ confidence interval and $5 \%$ marginal error; by adding $10 \%$ for none response the final sample size was 306 . First proportional allocation was made based on the number of pregnant mothers who were in ANC, and then systematic sampling was used to select study participants

\section{Data collection procedure and Measurements}

Socio-economic and demographic factors, pregnancy and maternal clinical characteristics were collected from all selected participants using a pre-tested interview administered semi-structured questionnaire as well as dietary intake information was collected using standard of World and Health Organization or Food and Agriculture Organization (WHO/FAO) classification guideline of nutritional intake and adapted to containing local food items to assess women dietary diversity. The respondents were asked to report the number of food in the past week they consumed into categories of the listed food items starting from prior of interviewed. In this study revealed 33 locally ate food varieties consumption of pregnant women within a week, used to construct the women dietary diversity Score (WDDS) then according to WHO/FAO guideline all food items merged to ten food groups these are; cereals; egg; milk and milk product; other vitamin A rich fruits, tubers and vegetables; dark green leafy vegetables; meat, poultry and fish; nut and seeds; other vegetables; other fruits and pulse or legumes [14].Consumers of a food item were defined as follows: intake of the food varieties at least once in the past week, regardless of the frequency, women that consumed a food varieties at least once in a week was scored as one or yes; in the current study the minimum and maximum food groups were three and nine respectively; WDDS was constructed as the sum of numbers of food groups consumed over the past week; dietary diversity score is classified as low, medium and high based on the women food groups consumed; higher score indicates higher diversity, as more food groups were eaten. There is no conventional cut-offs to indicate adequate or inadequate dietary diversity within a week for the women; hence, the score is converted into tertiles and the higher tertile of the score was taken as high or adequate women dietary diversity score and the middle and low tertiles were merged together and considered as low or inadequate women dietary diversity score.

\section{Data Processing and Analysis}

Data were cleaned and checked for consistencies as well as completeness and entered to EpiData version 3.5.1 and exported to SPSS version 20 for analysis. Descriptive and binary logistic regression analysis were done to see the association between the dependent and predictive variables in bivariate analysis then those variables which have statistically significant results ( $p$-value $<0.25$ ) were entered in to multivariable logistic regression model to identify the independent effect of each predictive variable on the outcome variable, finally the variables which have $p<0.05$ are noted significant with dietary diversity score.

\section{Result}


From this study, a total of 303 participants were involved with $99 \%$ response rate. The mean age of the attendants was $28.3 \pm 2.7$. Almost all (98\%) of the respondents were married, nearly two third $(68.6 \%)$ of the respondents were Hadiya. Only $24.1 \%$ of the respondents were can't read and write, $239(78.9 \%)$ of the women were house wife as well as around one fifth $(23.1 \%)$ of the respondents had less than 500 Ethiopia birr for monthly family members expenditure (Table 1).

\section{Pregnancy and Maternal clinical related Characteristics}

The mean age of first delivery of the participants was $20.7 \pm 1.7$ month. More than two third of respondents (78.9\%) had previous pregnancy from those $58.7 \%$ of them delivered at health facility while the rest delivered at home. This study revealed closely one third of the participants 98 (32.3\%) had two ANC visits plus with $45.2 \%$ of respondents were in the third trimester. The participants were also evaluated for whether they had history of some morbidity such as malaria, HIV/AIDS, Hypertension, diabetes mellitus, tuberculosis and intestinal helminthes during this study. Almost one fifth $(19.5 \%)$ of respondents were reported history of malaria, intestinal helminthes and hypertension in the last one year; however, none of the respondent was diagnosed to have any of the rest major morbidity during the current pregnancy (Table 2).

\section{Description of Nutritional Related Characteristics}

This study revealed only thirty (9.9\%) of the women have got information regarding nutrition such as iron source food, additional meal and healthy diets during pregnancy from different sources. The mean of twenty four hours meal intake frequency of the respondents was $2.7 \pm 0.52$. The mean of food variety consumed within a week of the respondents was $19.4 \pm 3.6$. From this study less than half of the participants have eaten milk and milk products; whole milk(46.2\%), cheese $(55.8 \%)$, yoghurt(22\%), butter(19.3\%); Cereals; teff(99.2\%), wheat(94.6\%), barley(16.8\%), millet (3.2\%), oats $(13.5 \%)$, rice(28.3\%); Dark green leafy vegetables; kale(16.9\%), Swiss chard (11.6\%), endive(14.3\%), lettuce(40.5\%); Other vitamin A rich fruit, tubers and vegetables; mango(32\%), papaya(22.5\%), carrots(24.4\%), potato (82.3\%); Other fruits; orange(38.6\%), lemon(11.2\%); Other vegetables; tomatoes(81\%), chilies(40.1\%); Meat, poultry and fish; beef(20.2\%), mutton(10\%), goat(2.6\%), poultry (1.6\%) and fish ( $0.8 \%)$; Pulse or Legumes; lentils $(92 \%)$, peas ( $87 \%)$, chicken peas $(22 \%)$, horse bean(33\%), kidney bean $(4.2 \%)$ and lastly $78 \%$ of the participants took products of inset (Table 3 and 4 ).

\section{Women dietary diversity score}

Out of ten food group consumption of the seven days, the mean of dietary diversity was $7 \pm 1.3$ with scores ranging from 3 to 9 food groups. Based on the score of tertiles almost half of the respondents ( $48.2 \%$ ) were in the medium ( $5-7$ food groups), $9.2 \%$ in low ( $<5$ food groups) and $42.6 \%$ had high dietary diversity ( $\geq 8$ food groups). There is no standard cut-offs to specify adequate or inadequate dietary diversity within a week for the women we used dietary diversity score by converting tertiles, according to this study 129 (42.6\%) of study participants have adequate dietary diversity score or had greater than or 
equal to eight food groups but 174 (57.4\%) consumed inadequate dietary diversity that is less than eight food groups within a week (Table 3 and Figure 1).

\section{Factors Associated with Dietary diversity among Pregnant Women}

In this study, both bivariate and multivariable logistic regression analysis were computed, In bivariate analysis, education of women, occupation of the women, gap between the previous and current pregnancy, history of health education about nutrition during pregnancy, monthly income of the family, number of family and frequency of meal were identified as the candidate for multivariable logistic regression analysis; in multivariable analysis revealed that; women who attended higher institution had 2.1 higher odds of attaining adequate dietary diversity as compared to those who can't read and write $(A O R=2.1 ; \mathrm{Cl}: 4.8,15.2)$, women whose family monthly income earned greater than 2000 Ethiopia birr 1.9 times (AOR=1.9; $95 \% \mathrm{Cl}: 1.06,7.1)$ more likely to have adequate dietary diversity as compared to those less than 500 Ethiopia birr, in addition women who got health education about nutrition concerning eating additional meals, iron source and diversified foods during pregnancy (AOR=2.3; 95\% Cl: 1.2, 4.4) were 2.3 times more likely to have adequate dietary diversity as compared to those who didn't get the information; furthermore, women's frequency of meal 3 times per day 8.3 times ( $\mathrm{AOR}=8.3 ; 95 \% \mathrm{Cl}: 4.5,15.6)$ and $\geq 4$ times (AOR=12.9; 95\% Cl: 3.1, 52) were 12.9 times had greater odds of achieving adequate dietary diversity than who had 2 times meal frequency per day (Table 5)

\section{Discussion}

Nutritional necessity increase during pregnancy so appropriate consumption of diversified diets is significant for maternal welfare as well as the growth, development and entire health of the fetus. The most important aim of determining dietary diversity is to encourage women to eat diversified diets rather than eating monotonous food all over their pregnancy.

This study revealed almost all $98.4 \%$ of women consumed cereals while only $20.1 \%$ ate meat, poultry and fish food group. The mean of dietary diversity was shown as $7 \pm 1.3$, this finding relates with the study done in Kenya among pregnant women where a mean dietary diversity score of $6.84 \pm 1.46$ was recorded [15] but not related with study done in Pakistan and Mekele town which was shown $6.17 \pm 0.99,4.9$ respectively $[16,17]$. According to this study pregnant woman dietary diversity score low, medium and high were found to be $9.2 \%, 48.2 \%$ and $42.6 \%$ respectively; the amount of study participants with adequate dietary diversity in the current study was $42.6 \%$ which is similar with the study conducted in Addis Ababa $39 \%$ of have high dietary diversity [10] and South Africa showed that $8.1 \%$ low, $44.2 \%$ medium and $47.7 \%$ of the women stated high-level dietary diversity [18], but this finding inconsistency the studies conducted on Gondar 21.1\% low, 62.7\% medium and 16.2\% high and Malaysia 2.5\% low, 9.1\% medium and $88.4 \%$ of the participants have high dietary diversity $[19,20]$, this dissimilarity might be due to variations in socio-economic, geographic, seasonal variability, eating habit and other sociodemographic factors of women's found in diverse areas of the countries. 
This study revealed women who attended educational institution had greater odds of attaining adequate dietary diversity as compared to those can't read and write, this outcome is comparable with study done in Kenya, South Africa and India [15,18,21], this might be due to women who attained school may have a better awareness about healthy diet during pregnancy.

This study also shown that women whose household monthly income earned greater than 2000 Ethiopia birr are more likely to have adequate dietary diversity as compared to those less than 500 ; this result is consistent with study done in Kenya and India [15,21]. This consistency suggested due to women in low socioeconomic ranks are expected to have financial constraints to access, avail and afford diverse diets. In addition history of attending health education about importance nutrition during pregnancy was significantly associated with dietary diversity; women who got health education about nutrition concerning eating additional meals, iron source and diversified foods during pregnancy were 2.3 times more likely to have adequate dietary diversity as compared to those who didn't get the information, this is consistent with study conducted in Kenya [15]. This similarity might be due to women who have awareness of nutrition is believed to be wide-open to updated information about the health benefits of different food varieties through various media sources.

Furthermore, this study noted women's frequency of meal four time per day consumed is 12.9 times had greater odds of attaining adequate dietary diversity as compared to those women who had two time per day, this finding is related with another study in FinoteSelam, North Ethiopia[22]. This could be explained by the fact that increasing frequency of consuming food items than common eating pattern of the family for compensating the normal physiological demand which is used by the fetus and mother is one of the approaches to raise dietary diversity of women.

\section{Conclusion}

In this study $42.6 \%$ pregnant women noted to have adequate dietary diversity score in Hossana town. The study revealed that educational status of mother, health education about nutrition during pregnancy, monthly income of the family and frequency of meal had significance association with dietary diversity among pregnant women. Based on the result efforts should be

strengthened to improve economic and educational status of pregnant mothers similarly nutrition education plus rising meal frequency should be delivered during ANC follow-up. The limitation of this study are quantitative measurement of nutrients were not applied due to absence of dataset or amount used that afford information on the quantity of food consumed by pregnant women to add up as among ten food group; recall and/or social desirability bias while subjects were requested to give dietary information and monthly income; furthermore, the study was cross sectional and may not display disparities of dietary practices the women by different seasons of the years.

\section{List Of Abbreviations}


ANC: Antenatal care; FAO: Food and Agriculture Organization; WDDS: Women dietary diversity score; WHO: World Health Organization; AOR: Adjusted Odds Ratio; Cl: Confidence Interval; COR: Crude Odds Ratio; DDS: Dietary Diversity Score; FVS: Food Varieties Score; SPSS: Statistical Package for Social Sciences, AIDS: Acquired immunodeficiency syndrome; HIV: human immunodeficiency virus

\section{Declarations}

\section{Ethics approval and consent to participate}

This study was approved by the ethical review board of Jimma University and ethical review committee of Hossana College of health Sciences; then permission was received from Hossana town health unit and other relevant administrative officials. Finally, informed verbal consent was obtained from the study participants prior to the actual data collection process. The consent was received from the pregnant mothers and minors such as children were not involved in the study

\section{Consent for publication}

Not applicable

\section{Availability of data and material}

The dataset is available from the corresponding author on reasonable request. Therefore, please contact the corresponding author for the data requests in SPSS form

\section{Competing interests}

The authors declare that they have no conflict of interests.

\section{Funding}

Jimma University and Hossana college of Health Sciences funded for data collection tools and presented lap top for data entry, analysis and presentations of this study. Moreover, these institutions established a research committee to approve a research topic, participate in research design and data collection and analysis.

\section{Authors' contributions}

RD: wrote the research proposal, participated in data collection, analysis and wrote the manuscript, BZ, DH and DT: participated in data collection, analysis and reviewed the paper and DH prepared this manuscript for publication. All the authors read and approved the final version of the manuscript.

\section{Acknowledgment}

First of all we would like to thank Jimma University and Hossana College of Health Science for financial and material support. Second our heartfelt thanks go to Hossana town health unit and other relevant 
stakeholders in the town for their cooperation. Finally we would like to thank data collectors, supervisors as well as the respondents for their invaluable contribution to this study.

\section{References}

1. Ellen Muehlhoff, Anthony Bennett and Deirdre McMahon. Milk and dairy product in Human Nutrition, FAO of the United nations, Rome Italy, 2013: 28

2. Ruel M. Is dietary diversity an indicator of food security or dietary quality? A review of measurement issues and research needs, FCND Discussion Paper: J Health, 2004

3. Mary Arimond, LivElinTorheim, Doris Wiesmann, Maria Joseph and Alicia Carriquiry. Dietary diversity as a measure of women's diet quality in resource-poor areas, Bangladesh. Food and Nutrition Technical Assistance Project, J Nutri., 2008: 7

4. Ramakrishnan,U, B. Imhoff-Kunsch and R. Martorell. Maternal Nutrition Interventions to improve maternal, newborn and child health outcomes. Nestlé Nutrition Institute Workshop Series: Pediatric Program, J Nutri., 2014: 78

5. HaoyueGao, Caroline K. Stiller, VeronikaScherbaum, Hans KonradBiesalski, Qi Wang, Elizabeth Hormann and Anne C. Bellows, et al. Dietary Intake and Food Habits of Pregnant Women Residing in Urban and Rural Areas, China; J ClinNutri. 2013. 10/3390: 6-7

6. Gina Kennedy, Maria Regina Pedro, Chiara Seghieri, Guy Nantel, and IngeBrouwer, Dietary Diversity Score Is a Useful Indicator of Micronutrient Intake in Non-Breast-Feeding Filipino Children. JNutri., 2011, 26-27.

7. Celeste Marinda and de Bruyn. Dietary adequacy, variety and diversity and associated factors (anthropometry and socioeconomic status) in pregnant women attending the Bishop LavisMou in Cape Town; 2015. 78

8. Martin CL, Sotres-Alvarez D, Siega-Riz AM and Saaka, M. Maternal Dietary diversity and infant outcome of pregnant women, in Ghana. 2015. 8

9. Habte TY and Krawinkel M. Dietary Diversity Score: A Measure of Nutritional Adequacy or an Indicator of Healthy Diet, Institute of Human Nutrition, Giessen, Germany, Journal of Nutrition and Health Sciences. 2016, 3/3. 5-7

10. AbdulhalikWorkicho, TeferaBelachew, GarummaTolu, BeyeneWondafrash, Carl Lachat and Patrick Kolsteren, et al. Household dietary diversity and animal source food consumption in Ethiopia evidence from the 2011 welfare monitoring survey. BMC Public Health, 2016. 16(1192): 5-7.

11. Jemal A Haidar and Rebecca S Pobocik. Iron deficiency anemia is not a rare problem among women of reproductive ages in Ethiopia: a community based cross sectional study school of public health, Addis Ababa University Ethiopian health and nutrition. BMC blood dis. 2009. 10(7): 1471-1482.

12. TaddeseAlemuZerfu, MelakuUmeta and KaleabBaye. Dietary habits food taboos and perceptions towards weight gain during pregnancy in arsi, rural central Ethiopia. J Health PopulNutr. (2016) (35): 22-24. 
13. DireslgneMisker, BegosewMisker and GistaneAyele. House hold dietary diversity and associated factors in Mirab Abaya wereda Southern Ethiopia, insight Medical publishing Group, 2016, 13(4): page. 1

14. Marie Claude Dop. Guidelines for measuring dietary diversity of women (FAO-FANTA), Montpellier, France, Nutripass Nutrition research. 2016.15

15. Willy Kiboi, Judith Kimiywe and Peter Chege. Determinants of dietary diversity among pregnant women in Laikipia County, Kenya: BMC Nutrition, 2017; 3:12. 5-6

16. Fatima Ali, InayatThaver and Shahzad Ali.Assessment of dietary diversity and nutritional status among pregnant woman in Islamabad, Pakistan. Jour. PubMed. 2014. 26(4): 507-8.

17. Abrehet A, Melkie EY and Molla MW. Prevalence and associated factors of anemia among pregnant women of mekelle town. BMC Res., 2014. 7(888): 7-9.

18. Samukelisiwe Sthokozisiwe Madlala. The dietary diversity, household food security status and presence of depression in relation to pregnancy pattern of weight gain and infant birth weight, Pietermaritzburg, South Africa, 2017: 85

19. GirmaNega, MelkieEndris, DegnetTeferi, Amanuel Nana and Rigbe W/Michael. Dietary Diversity and Associated Factors among Households in South Gondar, Ethiopia, 2015. 21

20. ShamsulZainal, JayashreeArcot, SharifahHaron, LailyPaim, NorhasmahSulaiman and JariahMasud, et al. Food Variety and Dietary Diversity Scores to Understand the Food-Intake Pattern among Selected Household, Malaysia, Ecology of Food and Nutrition, 2012. 51:4. 278

21. AnuRammohan, SrinivasGoli, Deepti Singh and Uma Singh. Maternal Dietary Diversity and Risk of Low Birth Weight: Empirical Findings from India, J Nutr. 2017. 8

22. GetnetMekuria, YalewsewWubneh and TilahunTewabe. Household dietary diversity and Associated factor among resident of FinoteSelam town, Ethiopia. BMC Nutri. 2017, 3:28.4-5

\section{Tables}

Table 1: Socio-demographic and economic related factors of the study participants in Hossana town, South Ethiopia, $2018(n=303)$ 


\begin{tabular}{|c|c|c|c|}
\hline Variables & Categories & Frequency & Percent \\
\hline \multirow{4}{*}{ Age group (years) } & $<20$ & 2 & 0.7 \\
\hline & $20-25$ & 60 & 19.8 \\
\hline & $26-30$ & 154 & 50.3 \\
\hline & $\geq 31$ & 88 & 28.9 \\
\hline \multirow{2}{*}{ Marital status } & Unmarried & 6 & 2 \\
\hline & Married & 297 & 98 \\
\hline \multirow{5}{*}{ Ethnicity } & Kambata & 52 & 17.2 \\
\hline & Hadiya & 208 & 68.6 \\
\hline & Gurage & 13 & 4.3 \\
\hline & Amhara & 19 & 6.3 \\
\hline & Silti & 11 & 3.6 \\
\hline \multirow[t]{4}{*}{ Religion } & Protestant & 251 & 82.8 \\
\hline & Orthodox & 22 & 7.3 \\
\hline & Catholic & 4 & 1.3 \\
\hline & Muslim & 26 & 8.6 \\
\hline \multirow[t]{4}{*}{ Educational level } & Can't read and write & 73 & 24.1 \\
\hline & Primary level & 108 & 35.6 \\
\hline & Secondary level & 76 & 25.1 \\
\hline & Higher institution & 46 & 15.1 \\
\hline \multirow[t]{4}{*}{ Spouse education } & Can't read and write & 13 & 4.3 \\
\hline & Primary level & 51 & 16.8 \\
\hline & Secondary level & 92 & 30.4 \\
\hline & Higher institution & 147 & 48.5 \\
\hline \multirow[t]{4}{*}{ Women's occupation } & House wife & 239 & 78.9 \\
\hline & Merchant & 21 & 6.9 \\
\hline & Government employee & 35 & 11.6 \\
\hline & Students & 4 & 2.6 \\
\hline \multirow[t]{4}{*}{ Spouse occupation } & Daily laborer & 86 & 28.4 \\
\hline & Farmer & 18 & 5.9 \\
\hline & Merchant & 154 & 50.8 \\
\hline & Government employee & 45 & 14.9 \\
\hline \multirow[t]{2}{*}{ Number of family } & $\leq 4$ & 161 & 53.1 \\
\hline & $>4$ & 142 & 46.9 \\
\hline \multirow[t]{3}{*}{ Monthly income level } & $<500$ & 70 & 23.1 \\
\hline & $500-2000$ & 104 & 34.3 \\
\hline & $>2000$ & 129 & 42.6 \\
\hline
\end{tabular}

Table 2: Pregnancy and clinical related characteristics of study participants in Hossana town, South Ethiopia, $2018(\mathrm{n}=303)$ 


\begin{tabular}{llll} 
Characteristics & Category & Frequency & Percent \\
\hline Gap of pregnancy & < 2years & 109 & 36 \\
\cline { 2 - 4 } & 2-4years & 108 & 35.6 \\
\cline { 2 - 4 } Number of pregnancy & Primi-gravida & 22 & 7.3 \\
\cline { 2 - 4 } & Multi-gravida & 239 & 21.1 \\
\hline Place of previous delivery & Home & 61 & 78.9 \\
\cline { 2 - 4 } & Health institution & 178 & 20.1 \\
\hline Gestational age & $\leq 12$ week & 4 & 58.7 \\
& $13-24$ week & 47 & 1.3 \\
\hline Number of ANC visit & $\geq 25$ week & 252 & 15.5 \\
& $1-2$ times & 137 & 45.2 \\
& 3 times & 129 & 42.6 \\
\cline { 2 - 4 } & 4 times & 37 & 12.2 \\
\hline
\end{tabular}

Table 3: Dietary related characteristics of pregnant women in public health facilities in Hossana town, South Ethiopia, 2018 ( $\mathrm{n}=303$ )

Characteristics

\begin{tabular}{llll}
\hline Attended health education during pregnancy & Yes & 30 & 9.9 \\
\cline { 2 - 4 } & No & 273 & 90.1 \\
\hline \multirow{3}{*}{ Kinds of nutritional Health education } & Iron source food & 2 & 0.7 \\
\cline { 2 - 4 } & Additional meal & 14 & 4.6 \\
\cline { 2 - 4 } Frequency of eating & Healthy diets & 11 & 3.6 \\
\cline { 2 - 4 } & Folic acid tablets & 3 & 1 \\
\hline DDS & 2 times & 86 & 28.4 \\
\cline { 2 - 4 } & 3 times & 204 & 67.3 \\
\cline { 2 - 4 } & $\geq 4$ times & 13 & 4.3 \\
\hline \multirow{2}{*}{ FVS } & Low & 28 & 9.2 \\
\cline { 2 - 4 } & Medium & 146 & 48.2 \\
\cline { 2 - 4 } & High & 129 & 42.6 \\
\cline { 2 - 4 } & $<17$ & 99 & 32.7 \\
\cline { 2 - 4 } & $18-23$ & 104 & 34.3 \\
\cline { 2 - 4 } & $24-29$ & 91 & 30 \\
\cline { 2 - 4 } & $\geq 30$ & 9 & 3
\end{tabular}

Table 4: Food groups consumption patterns of pregnant women in public health facilities in Hossana town, South Ethiopia, 2018 ( $\mathrm{n}=303$ ). 


\begin{tabular}{lll} 
Food groups & Frequency & Percent \\
\hline Egg & 118 & 38.9 \\
\hline Other vitamin A rich fruit, tubers and vegetables & 210 & 69.3 \\
\hline Cereals & 298 & 98.4 \\
\hline Dark green leafy vegetables & 260 & 85.8 \\
\hline Milk and milk product & 122 & 40.3 \\
\hline Other fruits & 240 & 79.2 \\
\hline Other vegetables & 288 & 95.1 \\
\hline Meat, poultry and fish & 61 & 20.1 \\
\hline Nut and seeds & 16 & 5.3 \\
\hline Pulse or Legumes & 300 & 99
\end{tabular}

Table 5: Predictors of dietary diversity with socio-demographic and socio-economic, pregnancy plus clinical related characteristics and nutritional intake factors among pregnant women in public health facilities in Hossana town, South Ethiopia, 2018 ( $n=$ 303). 


\section{iracteristics}

icational level of men

men

:upation

J between pregnancy

thly income level

$500-2000$
$>2000$

nber of family

$\leq 4$
$>4$

rition education at gnancy

al frequency

Yes

No 1

2 times

3 times

$\geq 4$ times

write

institution

House wife

Government

employee

Students

$<$ 2years
2-4years

$>4$ years
COR (95 \% $\quad$ P

CI)

Can't read and 1

3.7(1.2,

12.8)

3.8(1.1,

13.4)

$9.4(2.3,37.9)$

1

$\begin{array}{lll}1 & 1 \\ 0.9(0.4,2.2) & 0.83 \quad 1.9\end{array}$

0.83

$1.9(0.15$,
$14.2)$

0.003 2.5(0.16, 21.6)

AOR (95\% P

CI)

1

$\begin{array}{llll}4(1.2,9.9) & 0.003 & \begin{array}{l}14.2) \\ 2.5(0.16,\end{array} & 0.511 \\ & 21.6) & \\ 0.5(0.2,2.2) & 0.343 & \begin{array}{l}1.7(0.04, \\ 47.3)\end{array} & 0.782 \\ & & \end{array}$

47.3)

$1 \quad 1$

$\begin{array}{llll}0.8(0.4,1.3) & 0.31 & 1.1(0.98, & 0.424\end{array}$ 1.07)

$\begin{array}{llll}2.3(0.8,6.7) & 0.126 & 1.7(0.73, & 0.362\end{array}$ $5,7)$

$1 \quad 1$

$\begin{array}{llll}1.2(0.5,2.8) & 0.749 & .07(0.04, & 0.985\end{array}$ 2.8)

$\begin{array}{llll}2.8(1.3,5.8) & 0.009 & 1.9(1.06, & 0.034\end{array}$ 7.1)

1

1.98(1.1, $\quad 0.042 \quad 1.4(0.08, \quad 0.526$ 3.8) 6.3)

$2.9(1.7,5.2)<\quad 2.3(1.2,4.4) \quad 0.013$ 0.001

1

1

10.1(5.5, < 8.3(4.4, < 18.6) $\quad 0.001$ 15.6) 0.001 13.3(3.3, < 12.9(3.1, < 53.8)

0.001

\section{Figures}




\section{Title}

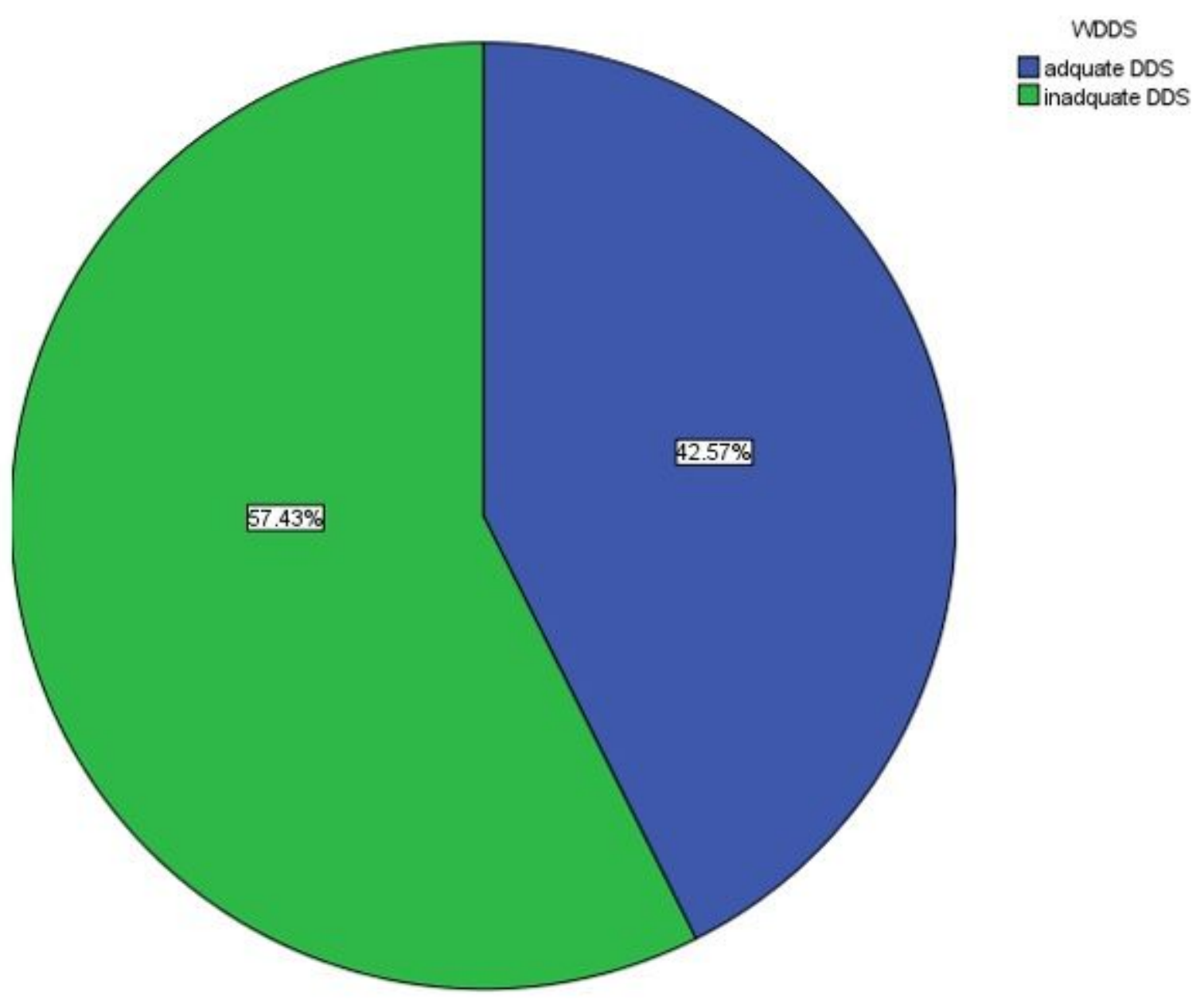

\section{Figure 1}

Prevalence of dietary diversity among pregnant women in public health facilities in Hossana town, South Ethiopia, $2018(n=303)$

\section{Supplementary Files}

This is a list of supplementary files associated with this preprint. Click to download.

- tables.pdf 